Hydrodynamic flow caused by active transport along cytoskeletal elements

This article has been downloaded from IOPscience. Please scroll down to see the full text article.

2007 EPL 7818001

(http://iopscience.iop.org/0295-5075/78/1/18001)

View the table of contents for this issue, or go to the journal homepage for more

Download details:

IP Address: 131.174.208.145

The article was downloaded on 11/07/2012 at 11:20

Please note that terms and conditions apply. 


\title{
Hydrodynamic flow caused by active transport along cytoskeletal elements
}

\author{
D. Houtman ${ }^{1}$, I. Pagonabarraga ${ }^{2}$, C. P. Lowe $^{1}$, A. Esseling-Ozdoba ${ }^{3}$, A. M. C. Emons ${ }^{3}$ and E. Eiser $^{1}$ \\ ${ }^{1}$ Van 't Hoff Institute for Molecular Sciences (HIMS), Universiteit van Amsterdam - Nieuwe Achtergracht 166 , \\ 1018 WV Amsterdam, The Netherlands \\ ${ }^{2}$ Departament de Física Fonamental, Universitat de Barcelona - Carrer Martí i Franqués 1, 08028-Barcelona, Spain \\ ${ }^{3}$ Wageningen University, Laboratory of Plant Cell Biology - Arboretumlaan 4, 6703 BD Wageningen, \\ The Netherlands
}

received 9 July 2006; accepted in final form 12 February 2007

published online 14 March 2007

PACS 87.16.Nn - Motor proteins (myosin, kinesin dynein)

PACS 67.40.Hf - Hydrodynamics in specific geometries, flow in narrow channels

PACS 05.40.-a - Fluctuation phenomena, random processes, noise, and Brownian motion

\begin{abstract}
We develop a simple lattice model to describe the hydrodynamic influence of active mass transport along bio-filaments on freely diffusing mass in the cell. To quantify the overall mass transport we include Brownian motion, excluded volume interactions, active transport along the filaments, and hydrodynamic interactions. The model shows that the hydrodynamic forces induced by molecular motors attached to the filaments give rise to a non-negligible flux close to the filament. This additional flux appears to have two effects. Depending on the degree of filament occupation it can exert a sufficiently large influence on unbound motors and cargo to modify their transport and also regulate the flux of motors bound to the filament. We expect such a mechanism is important in situations found in plant cells, where directional transport spans the entire cell. In particular, it can explain the cytoplasmic streaming observed in plant cells.
\end{abstract}

Copyright (c) EPLA, 2007

Introduction. - Molecular motors play a crucial role in the organization of cells and mass transport inside cells. Therefore, they are widely studied by biologists and more recently also by physicists. Although there are many different motors, each fulfilling a specific task, they all are proteins that generate motion by converting chemical energy, derived from hydrolysis of ATP, into mechanical work $[1,2]$. In this work we focus exclusively on processive molecular motors that, when attached to a transport filament, e.g. actin in plant cells, can bind to organelles or vesicles, and pull these along the filaments. Transport provided by molecular motors in this way is called "active transport". Thus, bio-filaments can be viewed as intercellular highways.

Intensive experimental work on various molecular motors has revealed the chemical and mechanical processes that an individual molecular motor undergoes in order to move along a filament. But for the understanding of the overall motion of motors and their cargo in cells, additional factors need considering. In particular, the viscous interaction with the surrounding medium and steric hindrance constitute two relevant mechanisms.
A third important process is thermal fluctuations because due to the motors' small size all unbound organelles in the cell behave like colloids, hence their motion is dominated by Brownian motion and is not ballistic. However, contrary to thermal motion, which does not produce directed motion, when a motor protein binds to a filament it will start moving on average with a directed motion. The polarity of the filament determines the direction of the motion for each type of motor.

The origin of the asymmetric motion of a motor on a filament is captured by simplified models, such as the Brownian ratchet model $[3,4]$. When a finite fraction of the motors move along a filament, one also needs to account for their interactions. To that end, it proves useful to disregard the details of how the filament-motor interaction generates a net displacement and describe it as an asymmetric simple exclusion process (ASEP). These models can be coupled to a Langmuir-type adsorption kinetics to account for the interaction between motors in the filament and in the surrounding suspension [5]. Such approaches have helped to elucidate the basic principles underlying cooperative motion of molecular motors and have been 
applied to analyze the relevance of strong confinement [5] and boundaries [6], which constitute important factors in highly crowded cells.

The interactions of the motors with the surrounding fluid can also have a deep influence in the collective behavior of molecular motors, although it has not been addressed before. Forces exerted by bio-filaments are usually in the range of picoNewtons [1], while the drag force exerted by water on a nanometer-size particle moving at $1 \mu \mathrm{m} / \mathrm{s}$ is of the order of $10^{-5} \mathrm{pN}$. Thus the hydrodynamic drag exerted on an isolated molecule is negligible, and it is small for organelles.

Despite this fact, we will show that these forces induce a qualitative change in the state of motion of suspended particles close to biofilaments, because the hydrodynamic forces generated when many active motors are present add up. As a result, moving bound motors can exert sufficient hydrodynamic force to give unbound motors in the vicinity a "free ride".

Besides the question of size, the relevance of hydrodynamic interactions together with excluded volume and crowding effects will also depend on the specific situation of interest. Collective hydrodynamic effects may be expected to play a minor role inside the complex threedimensional cytoskeletal network of animal cells. In this case the motion is only locally directed, on long (cell-like) length scales it is not. But in some situations with welldefined symmetry and boundaries, such as in neurons or plant cells, hydrodynamics may be relevant.

In particular, for cytoplasmic streaming in plant cells collective hydrodynamic effects could explain the rapid transport of organelles [7-10]. Here unidirectional active transport takes place trough cytoplasmic strands. Figure 1 shows a stamen hair cell of Tradescantia virginiana (spiderwort) where such strands are clearly visible, generating directional motion inside the cell. Microscopic observations have elucidated non-negligible flow in the cytoplasm caused by the movement of organelles [11].

Model. - In order to analyze the effects of solvent on the transport of bound and suspended motors, we have developed a lattice model that combines an asymmetric Brownian motion along the filament with positiondependent mobilities to include the effect of the interactions mediated by the surrounding fluid. It can be then regarded as an extension of ASEP models coupled to Langmuir adsorption kinetics in which hydrodynamic interactions are incorporated in a lattice description. We consider a lattice occupied by $N$ motor-organelle complexes. These complexes are chosen to be equal to each other in size, and have the same processivity and velocity. Biofilaments are added as linear segments connecting lattice sites, see fig. 2. The excluded volume of the motors is allowed for by forbidding two particles from occupying the same node simultaneously. Suspended motors diffuse freely, while bound motors perform an ASEP motion coupled with a Langmuir-type adsorption/desorption

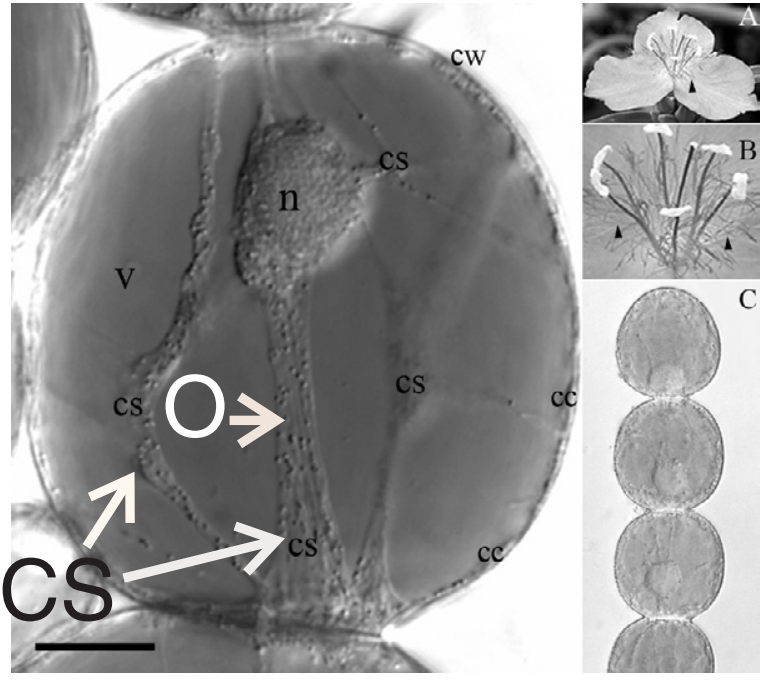

Fig. 1: Tradescantia virginiana (spiderwort) stamen hair cell. The cytoplasmic strands (CS) contain organelles (O) (white arrow); n: nucleus, v: vacuole, cc: cortical cytoplasm, cw: cell wall. Bar $=20 \mu \mathrm{m}$. Inserts: A) flower of T. virginiana, B) Magnification of stamens, arrowheads indicate stamen hairs, C) Single stamen hair.

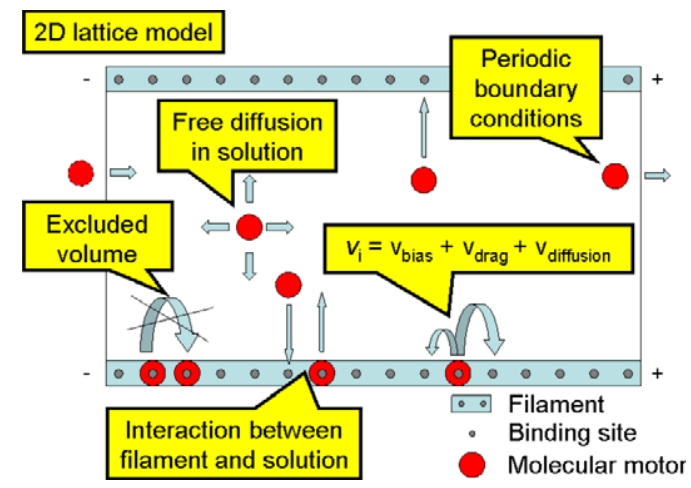

Fig. 2: Schematical view of the model. Shown is a 2D lattice consisting of cytoplasm embedded between two filaments. The possible moves of the molecular motors are shown.

kinetics. The model assumes the cytosol is Newtonian, which, to a first approximation, is reasonable for small motor/cargo velocities.

To include the hydrodynamic coupling between motors one must take into account that, due to the motors' small size and velocity, any fluid flow they induce will be at low Reynolds numbers ${ }^{1}$. As a result, the general fluid flow equation (the Navier-Stokes equations) simplifies to the linear Stokes equation,

$$
\eta_{s} \nabla^{2} \mathbf{V}-\nabla p=\sum_{k} \mathbf{F}_{k} \delta\left(\mathbf{r}-\mathbf{r}_{k}\right)
$$

\footnotetext{
${ }^{1}$ For organelles of a typical size $l \sim 1 \mu \mathrm{m}$ moving in water at a characteristic velocity $u \sim 1 \mu \mathrm{m} / \mathrm{s}$ have a Reynolds number, $R e=u l / \nu$, of order $R e \sim 10^{-6}$.
} 
Here $\eta_{s}$ is the viscosity of the solvent, $\nabla p$ the pressure difference, $\delta$ is a delta function, $\mathbf{r}_{k}$ is the position of the $k$-th particle and $\mathbf{V}$ the fluid velocity. The flow is generated by the force $\mathbf{F}_{k}$ that particle $k$ exerts on the fluid. This force is the sum of the effective force produced by the filament on the motor, $\mathbf{F}_{0}$, and the net force coming from motor-motor interactions. The fluid flow induces a drag on all the motors. As a result, the velocity of motor $i$ can generally be expressed as

$$
\mathbf{V}_{i}=\mu_{i i} \cdot \mathbf{F}_{i}+\sum_{j \neq i} \mu_{i j} \cdot \mathbf{F}_{i}+\mathbf{V}_{i}^{R}=\mathbf{V}_{0}+\mathbf{V}_{i, d}+\mathbf{V}_{i, r}
$$

where $j$ sums over all motors subject to a force, $\mathbf{V}_{i}=$ $\mu_{i i} \cdot \mathbf{F}_{i}=\mathbf{V}_{0}$ is the single motor velocity produced by the force $\mathbf{F}_{0}$ exerted by the filament (and it is hence nonzero only for bound motors), $\mathbf{V}_{i, r}$ is the thermal velocity and $\mathbf{V}_{i, d}$ corresponds to the hydrodynamic coupling. Due to the linearity of eq. (1), the last contribution is determined by the mobility matrix $\mu_{i j}$ which in the Oseen approximation reads [12]

$$
\mu_{i j}=\frac{3}{4} \mu_{i i} \frac{A}{r_{i j}}\left[\hat{\mathbf{I}}+\hat{\mathbf{r}}_{i j} \hat{\mathbf{r}}_{i j}\right], \quad i \neq j,
$$

where $A$ is the hydrodynamic radius of the particles, $\mu_{i i}=$ $1 /\left(6 \pi \eta_{s} A\right)$ the self-mobility, $\mathbf{r}_{i j}=\mathbf{r}_{i}-\mathbf{r}_{j}$, the distance between two motors and $\hat{\mathbf{r}}_{i j}=\mathbf{r}_{i j} / r_{i j}$ is a unit vector.

Equation (2) gives the dynamic equation which determines the motion of the motors. Since they move on the nodes on a lattice, we have implemented a lattice variant of the Ermak and McCammon method [13], taking into account that hydrodynamic interactions also introduce correlation in the motors' diffusion. In order to account for the hydrodynamic coupling, it is necessary to estimate the interparticle forces associated with excluded volume; we do it on the basis of the velocity change of the motor.

Finally, the biofilament processivity is accounted for by allowing the motors to detach from the filament with a certain probability $\kappa_{u}$. Motors in solution close to the filament, in turn, can also attach to the filament with probability $\kappa_{b}$. Motor interchange between the filament and the embedding solution determines the ratio between the solution and filament volume fractions, $\phi_{s}$ and $\phi_{\mu}$, respectively. Assuming uniform concentrations along the microtubule and in the solution a mass flux balance predicts the steady state relationship between solution concentration and filament occupation, $\phi_{\mu}^{-1}=1+$ $\kappa_{u}\left(1-\phi_{s}\right) / \kappa_{b} \phi_{s}$. We have taken values of $\kappa_{u}$ and $\kappa_{b}$ to ensure the required filament and solution concentrations.

For the sake of simplicity, we neglect the effects that an additional solid substrate could have on the hydrodynamic interactions between suspended and attached motors; we leave such generalizations and refinement of the analysis to subsequent work. Consequently, our goal in this letter is to look at the qualitative differences that the presence of the solvent has on the dynamics of attached and suspended motors compared to the predictions of previous analysis

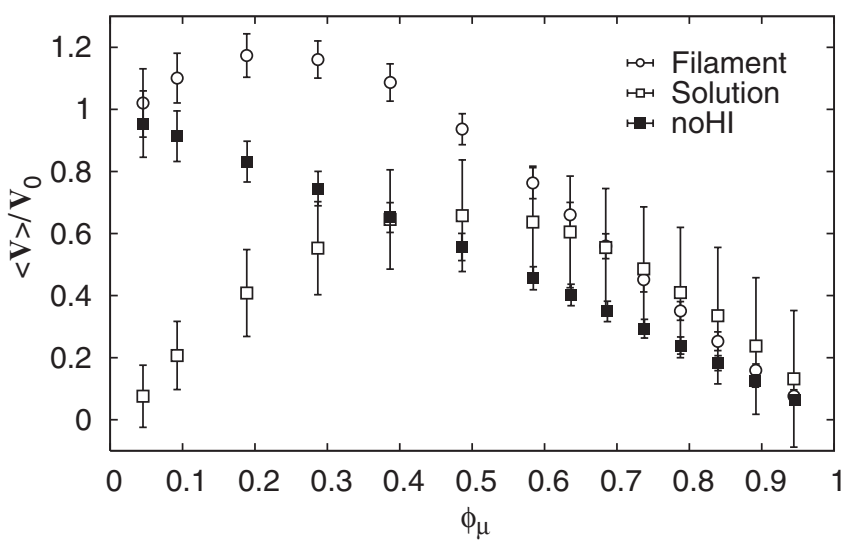

Fig. 3: The normalized velocity for different degrees of filament occupation $\phi_{\mu}$. The open circles refer to the filament, the open squares to the solution, the filled squares show data without hydrodynamic interactions.

that concentrated exclusively on transport in the absence of solvent.

Results. - We have considered the simplest geometry in which particles move in the two-dimensional plane confined between two filaments, although the hydrodynamic interactions correspond to those of a $3 \mathrm{D}$ fluid (we presume that the structure of the particles in the transverse direction can be neglected). Such a case can be regarded as a suspension of motors between substrates covered by a parellel set of filaments. Such an idealized example contains the basic dynamic couplings and facilitates the analysis. In order to analyze the interplay between activity, excluded volume and hydrodynamic forces, we fix the solution concentration to a small value, $\phi_{s}=0.05$ and analyze the collective behavior of the suspension + biofilament complex at different degrees of filament's occupation. In units of the lattice spacing, $l$, and simulation time step, $\Delta t$, for motors of unit mass we vary the force exerted by the filament between $1 / 2$ and 2 to control the single motor velocity, which should take values of the order of (but smaller than) a lattice spacing. Simulations are run for systems size $L$, containing around 1000 motors and for a few thousand time steps after thermalization. Within the Oseen description it is known that values of $A$ close to the particle radius may lead to numerical instabilities in configurations where particles are close to each other. To avoid such problems, and making use of the linearity of the system, we keep $A / l$ smaller than 1/5. For these parameters the motor Péclet number is of order one ${ }^{2}$. Nevertheless, the results we will discuss should not be severely affected by this fact, since we focus on mean collective motor velocities.

In fig. 3 we show the velocity at which motors move along a filament divided by the single motor velocity,

${ }^{2}$ For organelles of typical size $l \sim 1 \mu \mathrm{m}$ that move along a bio filament with a velocity $u \sim 1 \mu \mathrm{m} / \mathrm{s}$ and assuming a diffusion coefficient of $10^{-12} \mathrm{~m}^{2} / \mathrm{s}$ we find a peclet number of order $P e \sim 1$. 


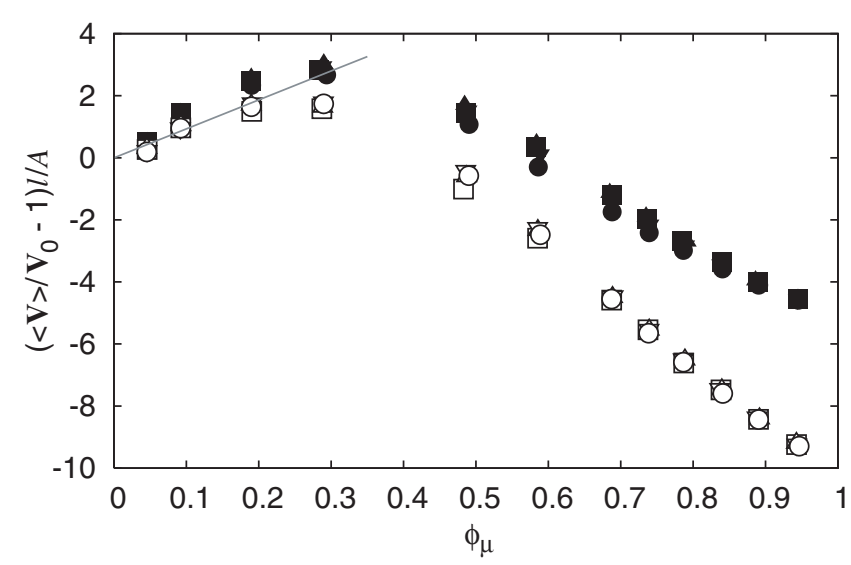

Fig. 4: Bound motor velocity for different filament occupation $\phi_{\mu} .\langle\mathbf{V}\rangle$ is the motor velocity, $\mathbf{V}_{0}$ the single motor velocity, $A$ the hydrodynamic radius and $l$ the lattice spacing. The line shows the theoretical expected increase in velocity due to HI (see text). $A$ and $\mathbf{V}_{0}$, expressed in lattice units as described in the main text, are varied: Open symbols $A=0.1 l$; filled symbols $A=0.2 l$; squares $\mathbf{V}_{0}=0.2$; triangles $\mathbf{V}_{0}=0.4$; inverted triangles $\mathbf{V}_{0}=0.6$; circles $\mathbf{V}_{0}=0.8$.

$\mathbf{V}_{0}$, as a function of the filament occupation, $\phi_{\mu}$. In the absence of hydrodynamic interactions (HI) the velocity decreases linearly with increasing occupation fraction due to excluded volume interactions.

When HI are considered, the drag first increases the overall bound motor velocity. At higher concentrations a second regime is achieved, where hindering due to excluded volume effects causes this velocity to decrease. Nevertheless, for all occupations the motors' velocity is larger than the corresponding one in the absence of HI. A second, qualitative, effect of the cooperativity induced by the solvent is displayed in the same figure where we show the average velocity of motors in solution. In the absence of HI particles can only display a net displacement along the filament. However, in the case with HI, there clearly exists a well-defined solution velocity which increases with $\phi_{\mu}$ until it reaches a maximum after which it decreases. The position of the maximum depends on the specific parameters considered. There seems to be an optimum filament occupation which is different for both the filament $\left(\phi_{\mu} \approx 0.2\right)$ and the solution $\left(\phi_{\mu} \approx 0.5\right)$. The position of these maxima seems to be insensitive for all simulation parameters explored (data not shown).

In fig. 4, we show the increase of the motors' velocity with respect to their biased velocity. Due to the linearity of the hydrodynamic coupling, in the regime where excluded volume interactions are negligible, the profiles are linear in $A$. Hence, different systems collapse in a single curve as a function of filament occupation. We can then use eq. (2) to estimate the initial increase in motors' velocity. If we rewrite it as

$$
\frac{\left(\mathbf{V}_{i}-\mathbf{V}_{0}\right) l}{\mathbf{V}_{0} A}=\frac{3}{4} \mu_{i i} \sum \frac{l}{r_{i j}}\left[\hat{\mathbf{I}}+\hat{\mathbf{r}}_{i j} \hat{\mathbf{r}}_{i j}\right], \quad i \neq j
$$

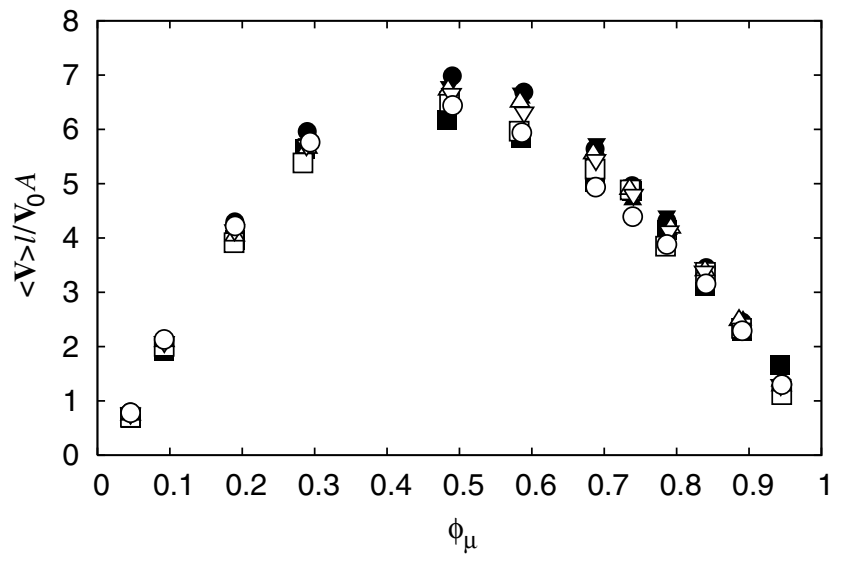

Fig. 5: Mean velocity $\langle\mathbf{V}\rangle$ in solution for different degrees of filament occupation $\phi_{\mu}$, hydrodynamic radius $A$, and single motor velocity $\mathbf{V}_{0}$ (expressed in lattice units as described in the main text), with $l$ being the lattice spacing. Open symbols $A=0.1 l$; filled symbols $A=0.2 l$; squares $\mathbf{V}_{0}=0.2$; triangles $\mathbf{V}_{0}=0.4$; inverted triangles $\mathbf{V}_{0}=0.6$; circles $\mathbf{V}_{0}=0.8$.

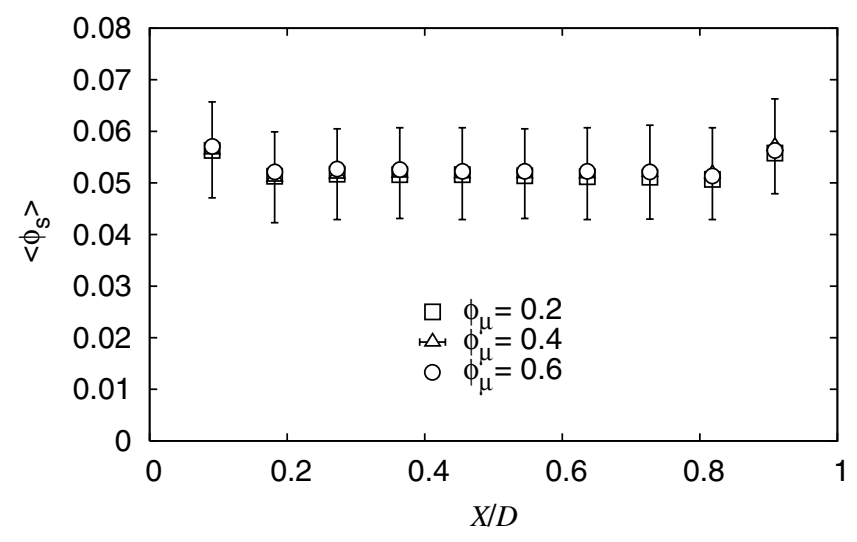

Fig. 6: Concentration profiles for unbound motors. $D$ is the distance between filaments, and $X$ the distance to the lower filament.

and approximate the right-hand side assuming a continuous and uniform distribution of motors, we get $\frac{\left(\mathbf{V}_{i}-\mathbf{V}_{0}\right) l}{\mathbf{V}_{0} A}=$ $\frac{3}{2} l \phi_{\mu} \ln \frac{L}{2 A}$, which agrees quantitatively with the simulation results. For total filament occupation, $\phi_{\mu}=1$ motors cannot move along the filament, $\langle V\rangle=0$.

In fig. 5 we display the mean velocity of unbound motors; these two plots show how the hydrodynamic coupling can be tuned by controlling the motors' size and biased velocity. Using a more realistic choice for the mobility tensor for particles at small separations (Brenner [14], Rotne-Prager [12]) this data does not change (data not shown), indicating that the mechanism described is generic and comes from the algebraic correlations induced by the embedding solvent.

Figure 6 shows the concentration of unbound motors across the width $D$ of the system. It shows that the interactions between the attached and suspended motors induce a uniform distribution of suspended motors, independently 


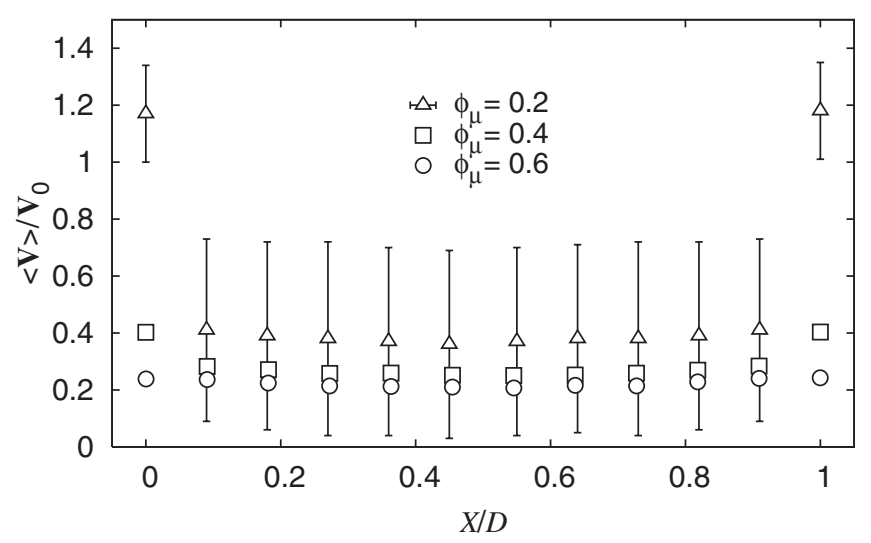

Fig. 7: Normalized velocity over the distance $D$ between the filaments for a distance $X$ from the lower filament for different biofilament occupation, $\phi_{\mu}$.

of filament occupation. One can clearly see that the hydrodynamic interactions have a minor effect on the concentration profile between the filaments. Also the velocity profile, as displayed in fig. 7 , shows that the velocity in solution is modified only in the filaments' neighbourhood.

Discussion. - We have shown that long-range collective hydrodynamic interactions lead to a substantial increase in the effective velocity of motors attached to a filament. Moreover, their motion leads also to net transport on the nearby unbound particles. This mechanism is not captured by models that consider only the activity and steric interactions of motors attached to biofilaments. Such an additional transport mechanism may be numerically as relevant as the mass transport obtained by direct motion of attached motors.

Due to its nature, this mechanism is more relevant for larger objects (suprananoscopic), for highly viscous environments and for transport on elongated geometries. One must take into account that as the dimensions grow simple diffusion becomes more inefficient as compared to convection.

The additive hydrodynamic force, as induced by the processivity of the filament, might not be large compared with the driving force which generates the motion of the attached motors but the cumulative effect can give rise to a net significant mass transport of the system. Obviously, such a constructive mechanism will be more prominent the more ordered the environment. In this sense, such a mechanism can be envisioned to be more important in situations as found in neurons or in cytoplasmic strands in plant cells. The outcome of our simulations suggests that this mechanism is indeed a plausible explanation for how cytoplasmic streaming really takes place.

$$
* * *
$$

IP thanks DGICYT of the Spanish Government and Distinció de la Generalitat de Catalunya (Spain) for financial support. We also thank D. FRENKEL for many helpful discussions.

\section{REFERENCES}

[1] Jonathan Howard, Mechanics of Motor Proteins and the Cytoskeleton (Sinauer Associates, Sunderland) 2001.

[2] Alberts B. et al., Molecular Biology of the Cell (Garland, New York) 2002.

[3] Austumian R. D., Science, 276 (1997) 917.

[4] Jülicher F., Ajdari A. and Prost J., Rev. Mod. Phys., 69 (1997) 1269.

[5] Lipowsky R., Klumpp S. and Nieuwenhuizen T. M., Phys. Rev. Lett., 87 (2001) 108101.

[6] Klumpp S. and Lipowsky R., J. Stat. Phys., 113 (2003) 233.

[7] DE Win A. H. N. et al., Cytometry, 29 (1997) 136.

[8] Pickard W. F., Plant, Cell Environ., 26 (2003) 1.

[9] Shimmen T. and Yokота E., Curr. Opin. Cell Biol., 16 (2004) 68.

[10] Sieberer B. and Emons A. M. C., Protoplasma, 214 (2000) 118.

[11] Esseling-Ozdoba A., Houtman D., van Lammeren A. A. M., Eiser E. and Emons A. M. C., in preparation.

[12] Dhont J. K. G., An Introduction to Dynamics of Colloids (Elsevier science B.V., Amsterdam) 1996, pp. 241-243, 250-253.

[13] Ermak D. L. and McCammon J. A., J. Chem. Phys., 69 (1978) 1352.

[14] Brenner M. P., Phys. Fluids, 11 (1999) 754. 